\title{
Cyclooxygenase-2 promotes tumor lymphangiogenesis and lymph node metastasis in oral squamous cell carcinoma
}

\author{
YOSHIHIRO MORITA $^{1,2}$, KENJI HATA ${ }^{1}$, MASAKO NAKANISHI ${ }^{1}$, TOSHIHIKO NISHISHO ${ }^{1}$, \\ YOSHIAKI YURA $^{2}$ and TOSHIYUKI YONEDA ${ }^{1}$ \\ Departments of ${ }^{1}$ Molecular and Cellular Biochemistry and ${ }^{2}$ Oral and Maxillofacial \\ Surgery II, Osaka University Graduate School of Dentistry, Osaka, Japan
}

Received February 22, 2012; Accepted May 16, 2012

DOI: 10.3892/ijo.2012.1529

\begin{abstract}
Oral squamous cell carcinoma (OSCC) is the sixth most common cancer and frequently metastasizes to the cervical lymph nodes, leading to poor survival of patients with OSCC. However, the mechanism of lymph node metastasis is not fully understood. To clarify the molecular mechanism underlying OSCC metastasis to regional lymph nodes, the highly metastatic fluorescent labeled OSCC cell line SAS-LM3 was successfully established allowing us to monitor the progression of lymph node metastases in a non-invasive manner. SAS-LM3 tumors showed increased lymphangiogenesis and elevated expression of VEGF-C, a potent stimulator of lymphangiogenesis, compared to parental SAS tumors. SAS-LM3 showed high expression of cyclooxygenase-2 (COX-2) compared to parental SAS cells and immunohistochemical analysis demonstrated intense COX-2 expression at the primary site. Inactivation of COX-2 by knockdown or the COX-2 inhibitor NS-398 decreased VEGF-C expression. Administration of COX-2 inhibitor NS-398 in SAS-LM3 tumor-bearing mice suppressed tumor lymphangiogenesis and lymphatic metastases. Collectively, our results indicate that COX-2 promotes tumor lymphangiogenesis and lymph node metastasis of OSCC. COX-2 ablation holds promise as a potential therapeutic approach for lymph node metastasis in OSCC.
\end{abstract}

\section{Introduction}

Oral squamous cell carcinoma (OSCC) is the sixth most common type of cancer worldwide: a total of 36,540 people in

Correspondence to: Dr Toshiyuki Yoneda, Department of Molecular and Cellular Biochemistry, Osaka University Graduate School of Dentistry, 1-8 Yamadaoka, Suita, Osaka 565-0871, Japan E-mail: tyoneda@dent.osaka-u.ac.jp

Abbreviations: COX-2, cyclooxygenase-2; OSCC, oral squamous cell carcinoma; VEGF-C, vascular endothelial growth factor-C; LYVE-1, lymphatic vessel endothelial hyaluronan receptor-1

Key words: oral squamous cell carcinoma, lymph node metastasis, lymphangiogenesis, cyclooxygenase-2 the United States are affected annually (1). The most common sites for early OSCC lesions are the ventral aspect of the tongue and the floor of the mouth in the oral cavity $(1,2)$. Although the diagnosis and surgical treatment of OSCC have advanced, survival rates of OSCC patients have not improved over the last 30 years, and only $40-50 \%$ of patients will survive for 5 years $(1,3)$. Moreover, since OSCC arises in the oral cavity, pharynx or larynx, it can cause severe dysfunction in swallowing, speech, and physical appearance, markedly decreasing the quality of life (QOL).

It has been well recognized that lymph node metastasis strongly correlates with the risk to survival in patients with OSCC. The incidence of neck lymph node metastasis in oral carcinoma varies from $25-65 \%(1,3)$. Although patients without lymph node metastases have a cumulative survival rate of approximately $50-70 \%$, this rate drops drastically to $30-50 \%$ in patients with documented nodal metastases $(4,5)$. Thus, understanding the pathophysiology of lymph node metastasis of OSCC is important for early diagnosis and treatment. However, precise molecular mechanisms of lymph node metastasis have not been elucidated, partly due to the lack of consistent and reproducible animal models.

The formation of new lymphatic vessels, lymphangiogenesis, is a critical step during the development of lymph node metastasis. Two major lymphangiogenic factors, namely vascular endothelial growth factor-C (VEGF-C) and -D, have been identified, and are linked to the promotion of lymphangiogenesis in animal models (6-8). In addition, the increase in lymphatic vessel density by VEGF-C greatly facilitates the spreading of tumor cells to lymph nodes (9). The occurrence of lymphangiogenesis can be detected using several lymphatic vessel-specific markers, such as VEGF receptor (VEGFR)-3, LYVE-1, Prox-1, podoplanin, and desmoplakin (10). VEGF-C and -D bind to the receptor VEGFR-3, and induce proliferation of lymphatic endothelial cells in vitro and lymphangiogenesis in vivo through the mitogen-activated protein kinase and phosphatidylinositol 3-kinase signaling pathways (11-14). Importantly, ablation of the VEGF-R3 mediated pathway by neutralizing antibodies against VEGF-R3 has been shown to inhibit lymph node metastasis $(12,13,15)$. Although the elevation of VEGF-C expression strongly correlates with the formation of metastases in regional lymph nodes in human thyroid, prostate, gastric, colorectal, breast, melanoma, and lung carcinoma (16-18), the pathological 
role of VEGF-C and VEGF-D in lymph node metastasis of OSCC is still unclear.

Cyclooxygenase-2 (COX-2), the inducible form of the COX enzymes (19), catalyzes the synthesis of prostaglandins with diverse biological activities, and its dysregulation plays a pivotal role in inflammation, tissue damage, and tumorigenesis $(20,21)$. The involvement of COX-2 in tumor activity is well documented: it is significantly increased in a range of human malignancies (22). Studies from transgenic animals have shown that the COX-2 gene is involved in the early stages of the oncogenic process of colorectal tumors (23). Moreover, a large amount of evidence points to a close association of $\mathrm{COX}$-2 upregulation with tumor invasion and metastasis in human colorectal, breast, and lung tumors $(24,25)$.

COX-2 has been implicated in several processes of cancer metastasis, especially angiogenesis, in which its major role is thought to be induction of the synthesis of prostanoids, which then stimulate the secretion of pro-angiogenic factors, including VEGF-A and fibroblast growth factor-2, from cancer cells and/or stromal fibroblasts $(26,27)$. In addition, COX-2 stimulates the proliferation, migration, and tube formation of vascular endothelial cells $(26,28)$. Several clinical studies have shown a correlation between the level of COX-2 expression and the extent of angiogenesis in cancer (29). Despite its evident importance in angiogenesis, the precise role of COX-2 in tumor lymphangiogenesis of OSCC remains poorly understood.

Here, we have successfully developed a model of OSCC that spontaneously metastases to the lymph nodes, enabling us to monitor the process using fluorescent-labeled human OSCC cells in a non-invasive manner. We found that elevated expression of VEGF-C under the control of COX-2 is critical for the development of lymphangiogenesis and lymph node metastasis and that inactivation of COX-2 clearly inhibited these metastatic properties in OSCC.

\section{Materials and methods}

Cell culture. Human OSCC SAS cells, which were originally isolated from the surgical specimens of a Japanese woman with a tongue primary lesion, were used in this study (30). The cells were cultured at $37^{\circ} \mathrm{C}$ under a $5 \% \mathrm{CO}_{2}$ atmosphere in Dulbecco's modified Eagle's medium (DMEM, Sigma, St. Louis, MO, USA) supplemented with $10 \%$ fetal bovine serum (FBS, Equitech-Bio Inc., Kerrville, TX, USA) and $100 \mu \mathrm{g} / \mathrm{ml}$ kanamycin (MeijiSeika, Tokyo). Cells were regularly certified free of mycoplasma contamination.

Animal model of lymph node metastasis. All experiments were conducted according to the ethical guidelines of the Institutional Review Boards, and approved by the Institutional Animal Use Committee of the Osaka University Graduate School of Dentistry.

Five-week-old, male, immunodeficient BALB/c nu/nu mice were anesthetized with pentobarbital $(0.05 \mathrm{mg} / \mathrm{g}$ body weight; Dainippon Pharmaceutical Co., Ltd.), and Venus-labeled SAS cells $\left(1 \times 10^{6}\right.$ in $0.1 \mathrm{ml}$ phosphate-buffered saline) were injected into the tongue (31). Metastatic lymph nodes could then be visualized by fluorescence stereoscopic microscopy (Leica Microsytems, Wetzlar, Germany).
To establish highly metastatic OSCC, tumor cells were isolated from metastatic lymph node lesions and re-inoculated after expansion in culture. Venus-positive SAS cells were purified by FACS Aria.

Lentiviral vector preparation and transductions. The lentiviral vector pLenti6/V5-Venus was constructed by subcloning PCR products of a Venus fragment into pLenti6/ V5 TOPO vector. Venus cDNA was kindly provided by Professor Atsushi Miyawaki (Riken, Japan). The vectors were packaged in 293FT cells using FuGENE 6 with ViraPower packaging mix (Invitrogen), and vector particles were harvested from the medium $49 \mathrm{~h}$ after transfection. SAS cells and vector particles were incubated with $6 \mu \mathrm{g} / \mathrm{ml}$ polybrene (Sigma) and Venus-labeled SAS cells were isolated by FACS Aria and cloned.

Immunohistochemistry. Mice were anesthetized with pentobarbital $(0.05 \mathrm{mg} / \mathrm{g}$ body weight) and fixed by perfusion with $4 \%$ paraformaldehyde in $0.1 \mathrm{M}$ phosphate buffer through the left cardiac ventricle. The tongues were removed and post-fixed for $24 \mathrm{~h}$, and then $7-\mu \mathrm{m}$ frozen sections were cut following a conventional method. Immunohistochemical staining of LYVE-1 (1:500 dilution; Abcam) and COX-2 (1:200 dilution; Cayman Chemical) were performed at $4^{\circ} \mathrm{C}$ overnight. As secondary antibodies, Alexa Fluor 555-conjugated antirabbit $\operatorname{IgG}$ (1:500; Invitrogen) were incubated for $1 \mathrm{~h}$ at room temperature. The sections were coverslipped with Vectashield Hard Set mounting medium with DAPI (Vector Laboratories, Burlingame, CA).

In vitro wound healing assay. For the wound-healing assay, $1 \times 10^{5}$ SAS cells per well were plated in DMEM containing $10 \%$ FBS in $10 \mathrm{~cm}$ plates and incubated for $24 \mathrm{~h}$. After confirming that a complete monolayer had formed, the monolayers were wounded by scratching lines in them with a standard 200- $\mu$ l plastic tip. Migration and cell movement throughout the wound area was observed with a phase-contrast microscope after $24 \mathrm{~h}$. The distance that the cells had migrated was measured on the photograph.

Histomorphometric analysis oflymphangiogenesis. In the model of lymph node metastasis, lymphangiogenesis was evaluated by lymphatic vessel density using LYVE-1 antibodies according to previous reports (32). Briefly, five hotspots (fields with the highest vascular density) in tumor areas were photographed at magnification of 200x, and digital images of LYVE-1-positive lymphatic vessels were captured. Area densities (percentage of total tumor area) of lymphatic vessels were then calculated using ImageJ software (NIH, Bethesda, MD, USA).

RNA preparation and quantitative real-time polymerase chain reaction. Total RNA from SAS cells was extracted using the Total RNA isolation system (NucleoSpin RNAII; MachereyNagel GmbH \& Co., Germany). First-strand cDNAs were synthesized using the Prime Script 1st strand cDNA synthesis kit (Takara) with Oligo-dT primers. Quantitative real-time reverse transcription-PCR (qRT-PCR) analysis was performed using the SYBR Green PCR protocol and a 7300 Real-Time PCR system (Applied Biosystems, Branchburg, New Jersey, USA). SYBR 

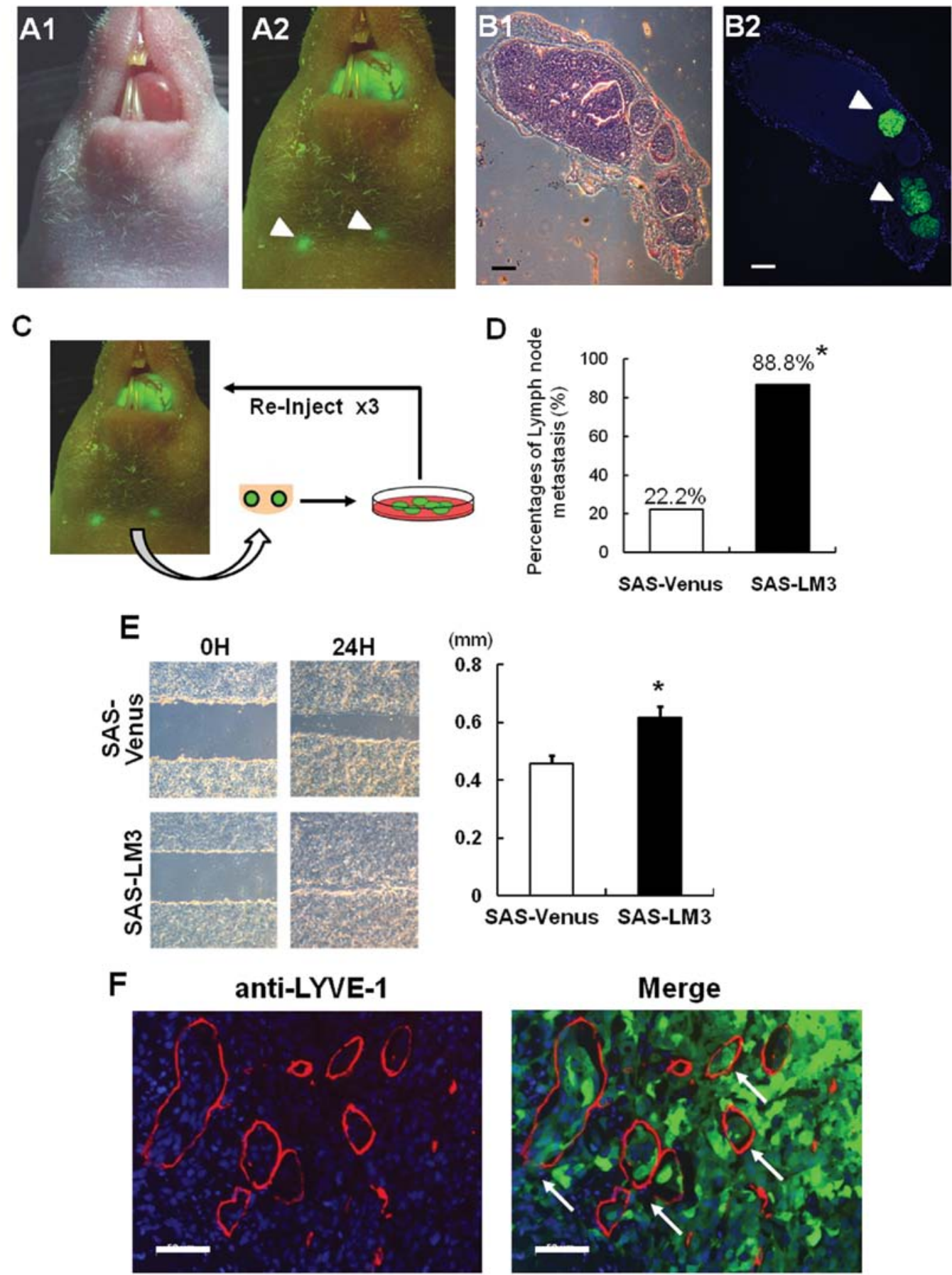

Figure 1. Model of OSCC lymph node metastasis and establishment of highly metastatic cells. (A) Representative macroscopic views and fluorescence images of lymph node metastasis. Bright field (A1) and dark field (A2) were taken 3 weeks after inoculation with Venus-labeled SAS cells. Cervical lymph node metastasis (arrowheads) was detected under the fluorescence stereomicroscope. (B) Representative H\&E-stained frozen sections (B1) and fluorescence images (B2) of lymph node metastasis from mice 3 weeks after inoculation of Venus-labeled SAS cells. Large cervical lymph node metastases are indicated by arrowheads. Scale bar, $200 \mu \mathrm{m}$. (C) Schematic representation of in vivo selection of highly metastatic cells. Venus-labeled SAS cells were inoculated into the tongue of nude mice. Cervical lymph node metastasis was detected under the fluorescence stereomicroscope 2 weeks after inoculation. Tumor cells were isolated from lymph nodes and re-inoculated after expansion in culture for three rounds. (D) The percentages of lymph node metastases generated by parental (SAS-Venus) and highly metastatic (SAS-LM3) cells. " $\mathrm{P}<0.01$. vs. SAS-Venus, $\mathrm{n}=9$ per group for each experiment. (E) SAS-Venus and SAS-LM3 cells were analyzed for cell migration using a wound-healing assay. Monolayers of SAS cells cultured in medium were scratched, and the distance between the edges of the scratches was measured $24 \mathrm{~h}$ after scratching. The data are expressed as the width filled with SAS cells (mean $\pm \mathrm{SD}$ ). ${ }^{*} \mathrm{P}<0.05$ vs. SAS-Venus cells. (F) Representative images assessing the localization of lymphatic vessels (red) and SAS-LM3 cells (green) in tumors. Note that abundant lymphatic vessels were observed in SAS-LM3 tumors, and cancer cells had invaded into peritumoral lymphatic vessels and spread along lymphatic vessels (arrows). Scale bar, $50 \mu \mathrm{m}$.

Green primers used for the amplification were as follows: human VEGF-C, sense 5'-GGAGGCTGGCAACATAACAG-3' and antisense 5'-ACGTCTTGCTGAGGTAGCTC-3'; human
VEGF-D, sense 5'-AGCGATCATCTCAGTCCACA-3' and antisense 5'-AGGTGCTGGTGTTCATACAG-3'; human COX-2, sense 5'-TGCATTCTTTGCCCAGCACT-3' and antisense 
A SAS-Venus
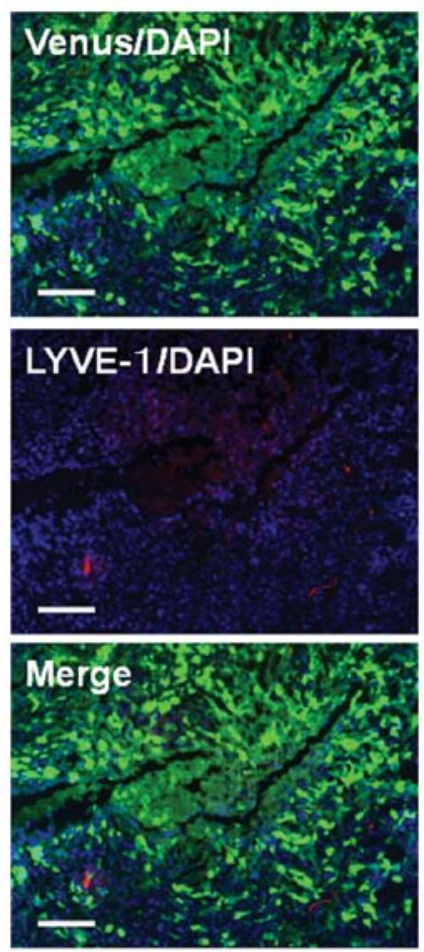

SAS-LM3
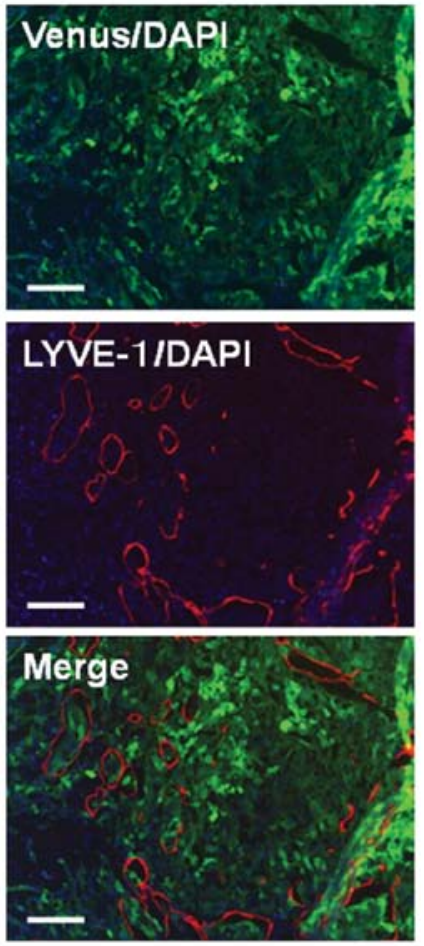

B

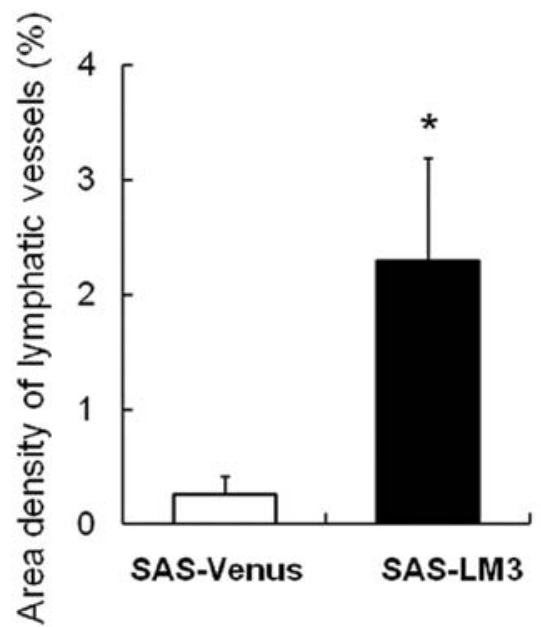

Figure 2. Enhanced lymphangiogenesis in highly metastatic SAS-LM3 tumor. (A) Immunohistochemical analyses of lymphatic vessels in orthotopic SAS-Venus or SAS-LM3 tumor (Venus: green). Frozen sections were stained for lymphatic vessels (anti-LYVE-1: red) and nuclei (DAPI: blue). Scale bar, 100 $\mu \mathrm{m}$. (B) Quantification of tumor lymphatic vessels. The data were expressed as mean area density of LYVE-1-positive pixels per microscopic field (mean \pm SD). ${ }^{*}<0.01$ vs. SAS-Venus.

5'-AAAGGCGCAGTTTACGCTGT-3'; human $\beta$-actin, sense 5'-AGCGGGAAATCGTGCGTG-3' and antisense 5'-CAGGGT ACATGGTGGTGGTGCC-3'. mRNA expression levels were normalized to that of $\beta$-actin.

Enzyme-linked immunosorbent assay (ELISA). SAS cells were seeded in 48-well plates and incubated in serum-free DMEM for $48 \mathrm{~h}$. The conditioned medium was collected, and human VEGF-C protein levels quantified using the Quantikine VEGF-C ELISA kit (R\&D Systems, Minneapolis, MN, USA) according to the manufacturer's instructions.

COX-2 inhibitor. NS-398(N-[2-(cyclohexyloxy)-4-nitrophenyl]methanesulfonamide), a COX-2 inhibitor, was purchased from Cayman Chemical (Ann Arbor, MI, USA).

Statistical analysis. The data are presented as the mean \pm SD, except morphometric analysis data, which are expressed as mean \pm SE. Student's t-test was used to compare data between two groups. P-values of $<0.05$ were considered to have statistical significance.

\section{Results}

Establishment of highly metastatic oral squamous cell carcinoma. To understand the underlying mechanism of lymph node metastasis of OSCC, we first aimed to establish a highly metastatic model of OSCC using fluorescent-labeled OSCC cell lines. We inoculated several OSCC cell types into mouse tongue, and found that SAS cells that had originally been isolated from oral cancer showed discernible tumorigenesis in vivo. We stably overexpressed Venus protein, an improved version of GFP, into SAS cells (SAS-Venus). Venus-labeled SAS cells spontaneously metastasized to the cervical lymph nodes 3 weeks after tongue inoculation, and fluorescent labeling enabled us to perform in vivo monitoring of lymph node metastasis under a fluorescence stereomicroscope (Fig. 1A). Histological examination also showed the metastasis of SAS-Venus cells to the lymph nodes (Fig. 1B). To obtain highly metastatic cells by in vivo selection, tumors were recovered from metastatic lesions, expanded in culture, and re-inoculated into mice (Fig. 1C). Finally, we established highly metastatic SAS cells (named SAS-LM3) after three rounds of in vivo selection using the poorly metastatic human cell line SAS-Venus as a starting point. The incidence of lymph node metastasis reached $88.8 \%$ in the fourth generation of the mouse model (Fig. 1D). In vitro wound healing assay revealed that migration activity was also increased in SAS-LM3 cells compared to SAS-Venus cells (Fig. 1E). Interestingly, immunohistochemical analysis using LYVE-1 antibody, a specific lymphatic vessel marker, demonstrated abundant lymph node vessels in SAS-LM3 tumor tissues (Fig. 1F). Moreover, we found that SAS-LM3 cancer cells in this model invaded into peri-tumoral lymphatic vessels and spread along lymphatic vessels toward the regional lymph nodes (Fig. 1F). These data suggest that SAS-LM3 cancer cells are a relevant preclinical animal cancer model for non-invasive imaging of lymph node metastases and the determination of underlying molecular mechanism. 
A

VEGF-C MRNA

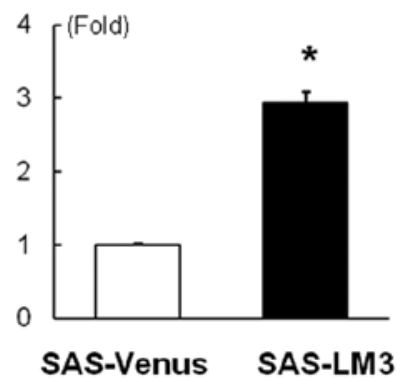

VEGF-D mRNA

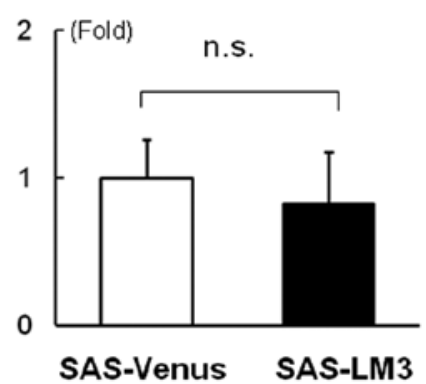

B

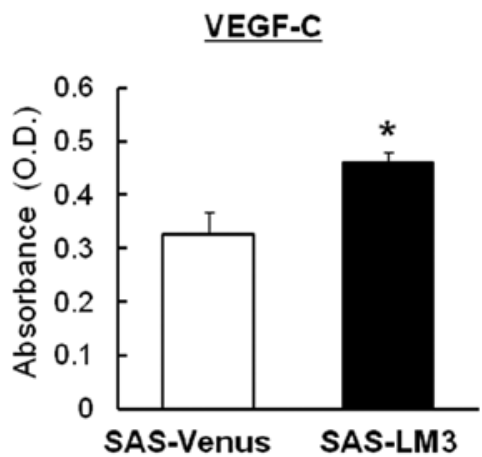

Figure 3. Increased VEGF-C mRNA expression and production in highly metastatic SAS-LM3 cells. (A) Total RNA isolated from SAS-Venus and SAS-LM3 cells was used for qRT-PCR analysis of VEGF-C and VEGF-D. The data are expressed as fold activation normalized to SAS-Venus (mean \pm SD). ${ }^{*}<0.05$ vs. SASVenus; n.s., not significant. (B) VEGF-C production in SAS-Venus and SAS-LM3 cells was determined by ELISA. The data are expressed as O.D. (mean \pm SD). ${ }^{*} \mathrm{P}<0.05$ vs. SAS-Venus.

Increased lymphangiogenesis and VEGF-C expression in SAS-LM3 tumors. Since abundant lymphangiogenesis was observed in SAS-LM3 tumors (Fig. 1G), we next quantified tumor lymphangiogenesis in SAS-Venus and SAS-LM3 tumors. Immunohistochemical staining of LYVE-1 revealed that the size and number of lymphatic vessels were dramatically increased in mice inoculated with SAS-LM3 cells compared with those inoculated with SAS-Venus cells (Fig. 2A). Increased lymphangiogenesis in SAS-LM3 tumors was validated by the quantification of LYVE-1-positive areas (Fig. 2B). These data raised the possibility that SAS-LM3 cells produce the lymphangiogenic growth factors VEGF-C and -D. We found that mRNA expression of VEGF-C, but not VEGF-D, was elevated in SAS-LM3 cells compared with SAS-Venus cells (Fig. 3A). VEGF-C production in SAS-LM3 cells was significantly higher than in SAS-Venus cells (Fig. 3B). These data suggest that elevated expression of VEGF-C seems to be an important feature of highly metastatic OSCC.

Involvement of $\mathrm{COX}-2$ in tumor lymphangiogenesis and lymph node metastasis of OSCC. Recent studies have suggested that COX-2 is involved in lymphangiogenesis (32). Accordingly, we examined whether expression of COX-2 was associated with the increase in VEGF-C expression and lymphangiogenesis in SAS-LM3 cells. To approach this, we first compared COX-2 expression in SAS-LM3 and SAS-Venus cells, and found that COX-2 mRNA expression was elevated in SAS-LM3 compared with SAS-Venus cells (Fig. 4A). Immunohistochemical staining detected COX-2 protein in Venus-positive OSCC inoculated in mice (Fig. 4B). To confirm the direct effects of COX-2 on VEGF-C expression in SAS-LM3 cells, we investigated the effect of $C O X-2$ knockdown on VEGF-C expression. We confirmed that $C O X-2$ shRNA decreased COX-2 expression at the protein level (Fig. 4C). Importantly, knockdown of $C O X-2$ significantly decreased the expression of VEGF-C mRNA in SAS-LM3 cells (Fig. 4D). Moreover, the COX-2-selective inhibitor NS-398 also decreased VEGF-C mRNA expression (Fig. 4E) and production (Fig. 4F).

To further clarify the role of COX-2 in lymph node metastasis of OSCC, we subsequently investigated the effect of
NS-398 on tumor lymphangiogenesis in our animal model. As shown in Fig. 5A, tumor lymphangiogenesis was significantly decreased in NS-398-treated mice compared with control mice. Quantification of LYVE-1-positive areas in hotspots confirmed these findings (Fig. 5B). Importantly, NS-398 significantly suppressed the rate of lymph node metastasis (Fig. 5C and D). These data suggest that COX-2 contributes to the development of lymphangiogenesis and lymph node metastasis of OSCC.

\section{Discussion}

Cervical lymph node metastasis significantly correlates with poor survival in patients with OSCC. A better understanding of the molecular mechanisms underlying lymph node metastasis is important for the early diagnosis and development of effective treatments of OSCC, which in turn leads to improved QOL and survival. However, little attention has been paid to the understanding of the pathophysiology of OSCC lymph node metastasis. Here, we have established an animal model of OSCC lymph node metastasis in an attempt to uncover its mechanism. We generated a highly metastatic OSCC cell line, SAS-LM3, which exhibited enhanced lymphangiogenesis. We demonstrated that increased VEGF-C under the control of COX-2 is critical for lymph node metastasis, and that inhibition of COX-2 clearly decreased lymphangiogenesis and lymph node metastases. Our results suggest that $\mathrm{COX}-2$ is involved in lymphangiogenesis and lymph node metastases, and thus is a potential therapeutic target in the treatment of OSCC.

COX-2 expression has been reported to be significantly increased in a variety of human cancer cells. High expression of COX-2 is associated with tumor growth, apoptosis, angiogenesis and metastasis (33-36). Previous studies have also shown that blockage of the COX-2 pathway is a promising antitumor strategy, and COX-2 inhibitors have potential as chemopreventive agents in OSCC $(37,38)$. Our preclinical studies clearly showed COX-2 to be critical for tumor lymphangiogenesis and lymph node metastasis of OSCC. Although previous clinical studies described a correlation between COX-2 expression and lymph node metastasis in tumors including gastric (39), lung (40), breast (41) and prostate (42), 


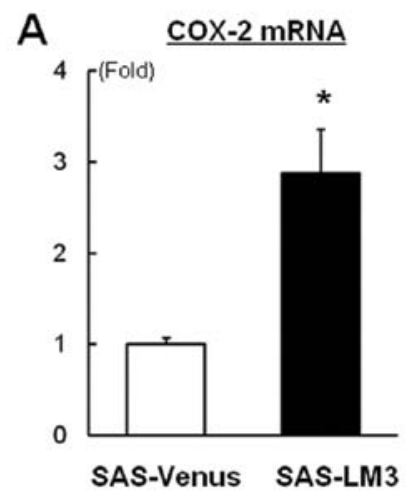

B
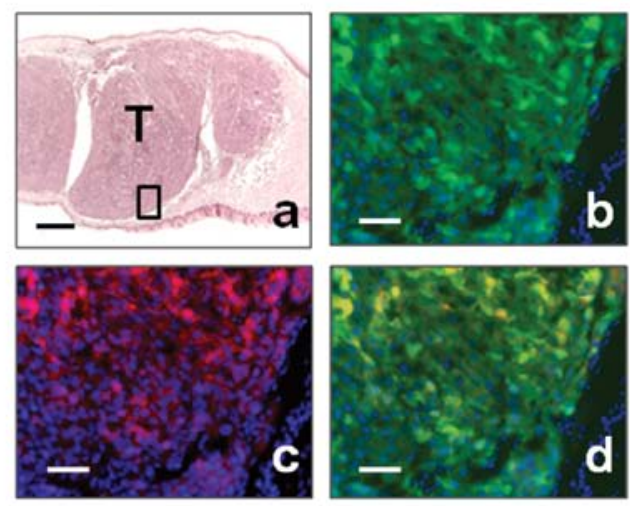

C
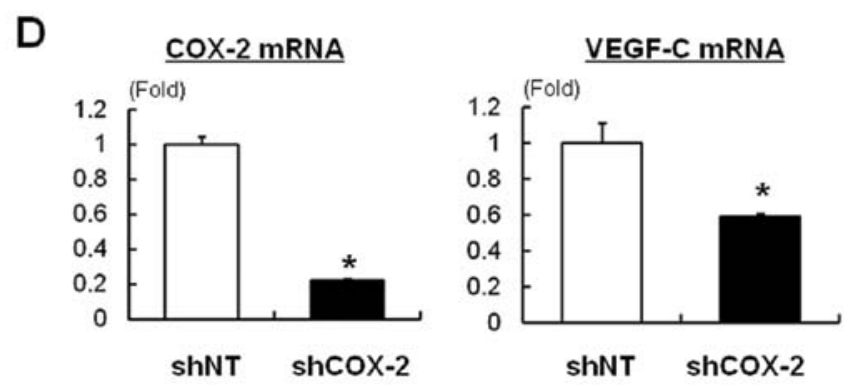

E

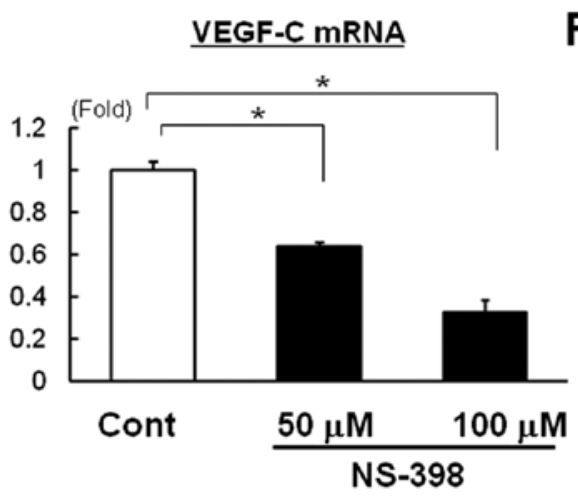

$\mathbf{F}$

VEGF-C

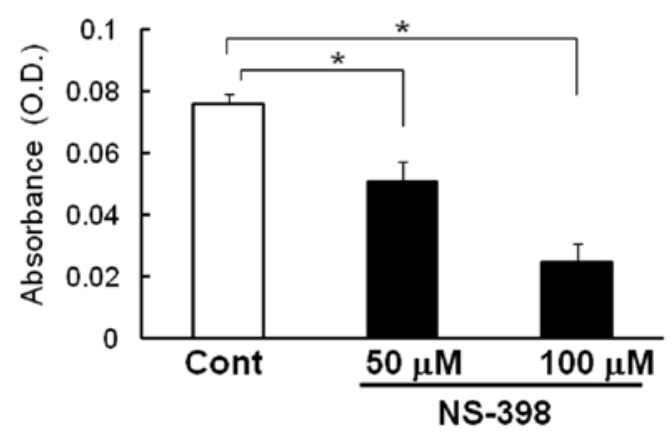

Figure 4. Increased COX-2 expression in highly metastatic SAS-LM3 cells is associated with VEGF-C expression. (A) Total RNA isolated from SAS-Venus and SAS-LM3 cells was used for qRT-PCR analysis of COX-2. The data are expressed as fold activation normalized to SAS-Venus (mean \pm SD). "P<0.05 vs. SASVenus. (B) Immunohistochemical analyses of COX-2 in SAS-LM3 tumors. H\&E staining of frozen sections of SAS-LM3 tumor (a) and the higher magnification of the boxed area: (b) Venus-expressing SAS-LM3 cells (green), (c) immunostaining of COX-2 (red) and (d) merge. Scale bars, (a) $500 \mu \mathrm{m}$, (b-d), $50 \mu \mathrm{m}$. (C) Knockdown of COX-2 by shRNA. SAS-LM3 cells were transfected with shNT or shCOX-2 and the level of COX-2 protein was analyzed with western blotting. (D) Total RNA isolated from SAS-LM3 cells transfected with shCOX-2 or shNT was used for qRT-PCR analysis of COX-2 and VEGF-C. The data were expressed as fold activation normalized to shNT (mean $\pm \mathrm{SD}$ ). ${ }^{*} \mathrm{P}<0.05$ vs. shNT. (E) SAS-LM3 cells were treated with vehicle (Cont) or NS-398 for $48 \mathrm{~h}$ and total RNA isolated from SAS-LM3 cells were used for qRT-PCR analysis of VEGF-C. The data are expressed as fold activation normalized to SAS-Venus (mean \pm SD). ${ }^{*} \mathrm{P}<0.05$ vs. Cont. (F) VEGF-C production in SAS-LM3 cells treated with vehicle (Cont) and NS-398 was determined by ELISA. The data are expressed as O.D. (mean \pm SD). ${ }^{*} \mathrm{P}<0.05$ vs. Cont.

the role of COX-2 in tumor lymphangiogenesis and lymphatic metastasis in OSCC has remained poor. Here, we showed that highly metastatic OSCC exhibited augmented expression of COX-2, which significantly correlated with increased lymph node metastasis. Lymphangiogenesis is regulated by various growth factors including VEGF-C/D, transforming growth factor $\beta$ (TGF- $\beta$ ), platelet-derived growth factor and fibroblast growth factor 2 (FGF2) $(43,44)$, which can all be potential therapeutic targets. Recent studies suggest that inhibition of VEGF-C/D and their cognitive receptor VEGFR-3 is an alternative therapeutic approach for lymph node metastasis, and that neutralizing antibodies against VEGFR-3 inhibited lymph node metastasis $(12,13,45)$. Our results showing that
VEGF-C expression was highly correlated with lymphatic metastasis of OSCC suggest that therapeutic approaches targeting VEGFR-3 using neutralizing antibody could also be beneficial for patients with OSCC.

Recent studies have shown that the epithelial-mesenchymal transition (EMT) play important roles in the process of cancer metastasis (46). EMT is associated with the increased cellular motility which enables cancer cells to migrate into distant organs. Although various cytokines are reported to induce EMT in cancer cells, it is well established that TGF- $\beta$ is the major and potent inducer of EMT (47). TGF- $\beta$ activates Smad proteins and activated Smads regulate several genes including Snail and Twist which causes EMT (47). It is likely that EMT 
A
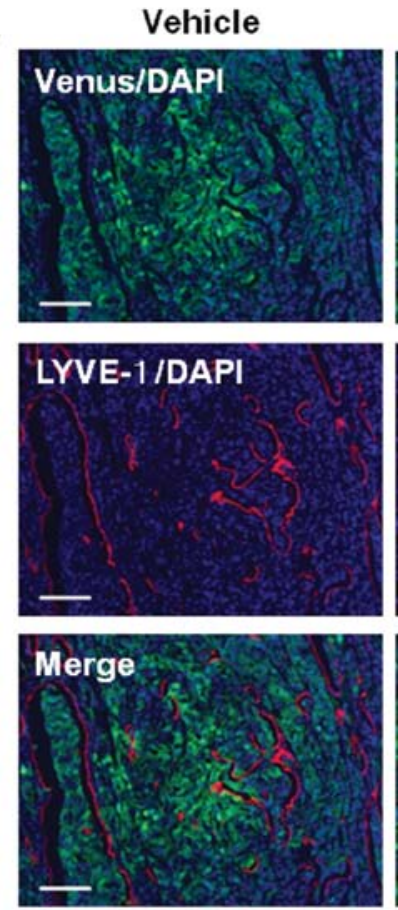

C

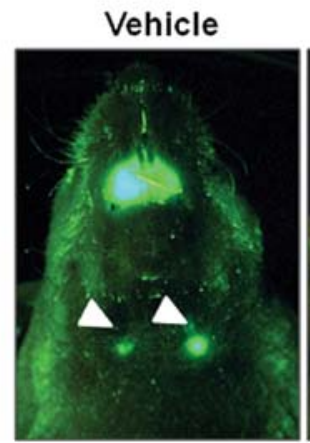

NS-398

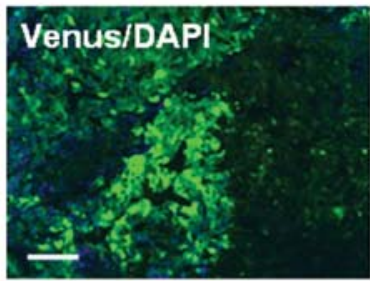

LYVE-1/DAPI

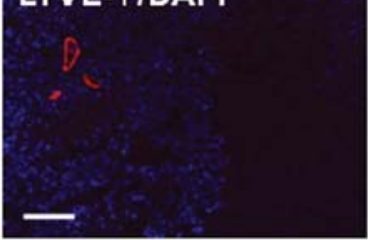

Merge

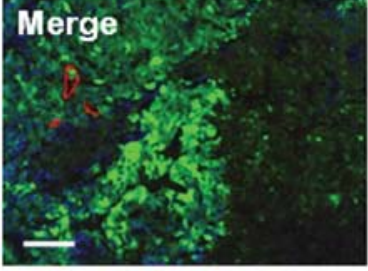

NS398

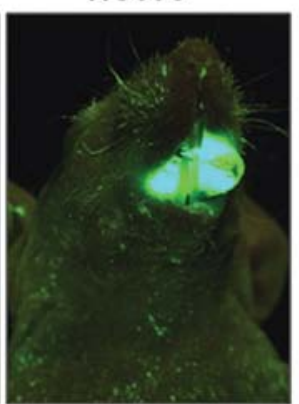

B

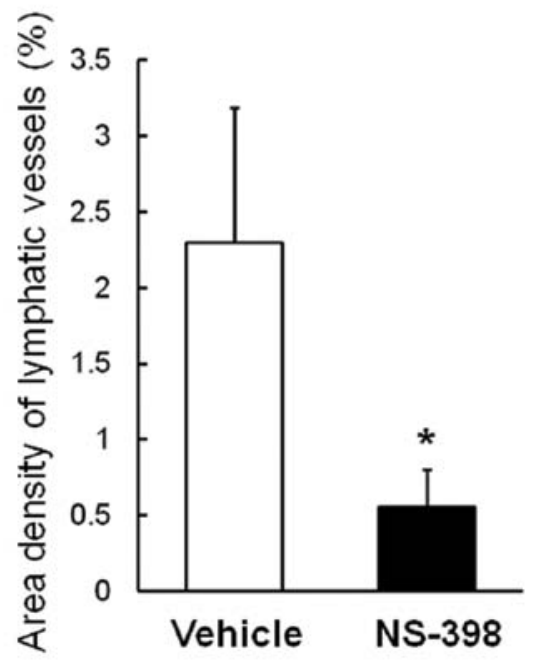

Figure 5. Effect of COX-2 inhibitor on lymphangiogenesis and lymph node metastasis in vivo. (A) Immunohistochemical analyses of lymphatic vessels in SASLM3 tumor treated with vehicle or NS-398 (Venus: green). Frozen sections were stained for lymphatic vessels (anti-LYVE-1: red) and nuclei (DAPI: blue). Scale bar, $100 \mu \mathrm{m}$. (B) Quantification of tumor lymphatic vessels. The data are expressed as mean area density of LYVE-1-positive pixels per microscopic field (mean \pm SD). ${ }^{*} \mathrm{P}<0.05$ vs. vehicle. (C) Representative fluorescence images of lymph node metastasis in SAS-LM3-bearing mice treated with vehicle or NS-398. Cervical lymph node metastasis (arrowheads) was detected under the fluorescence stereomicroscope. (D) The percentages of lymph node metastasis in SAS-LM3bearing mice treated with vehicle or NS-398 ( $\mathrm{n}=11$ per group for each experiment). ${ }^{*} \mathrm{P}<0.01$. vs. vehicle.

was upregulated in SAS-LM3 cells compared to SAS-Venus cells and therefore showed high metastatic activity. To support this notion, cellular motility of SAS-LM3 cells were increased (Fig. 1E) and SAS-LM3 cells showed high expression of fibronectin which was described as an acquired mesenchymal cell marker in EMT (data not shown). Further studies are needed to clarify the role of EMT in lymph node metastasis of OSCC.

In the present study, we focused on COX-2 as the regulator of lymphangiogenesis and lymph node metastasis of OSCC. However, various factors including signaling molecules, cytokines and enzymes should be involved in metastatic events. Additional studies using our cells established in this study would be useful to determine the precise molecular mechanisms responsible for the lymph node metastasis. For example, microarray analysis between SAS-Venus and SAS-LM3 may lead to the identification of novel regulator of lymphangiogenesis and non-invasive animal model of lymph node metastasis enable us to examine the effect of new therapeutic agents.

In conclusion, our results suggest that increased expression of COX-2 is critical for the development of lymphatic metastasis in OSCC. The results also suggest that COX-2 is a potential therapeutic target in designing pharmacologic interventions for the treatment of oral cancer.

\section{Acknowledgements}

We are grateful to Dr Riko Nishimura (Osaka University Graduate School of Dentistry) for helpful discussions. This study was supported by a Grant-in-Aid for Scientific Research 
on Priority Areas (TY) from the Ministry of Education, Culture, Sports, Science and Technology of Japan, and the 21st Century COE Program (TY).

\section{References}

1. Jemal A, Siegel R, Xu J and Ward E: Cancer statistics, 2010. CA Cancer J Clin 60: 277-300, 2010.

2. Hunter KD, Parkinson EK and Harrison PR: Profiling early head and neck cancer. Nat Rev Cancer 5: 127-135, 2005.

3. Forastiere A, Koch W, Trotti A and Sidransky D: Head and neck cancer. N Engl J Med 345: 1890-1900, 2001.

4. Gil Z, Carlson DL, Boyle JO, et al: Lymph node density is a significant predictor of outcome in patients with oral cancer. Cancer 115: 5700-5710, 2009.

5. Mamelle G, Pampurik J, Luboinski B, Lancar R, Lusinchi A and Bosq J: Lymph node prognostic factors in head and neck squamous cell carcinomas. Am J Surg 168: 494-498, 1994.

6. Joukov V, Pajusola K, Kaipainen A, et al: A novel vascular endothelial growth factor, VEGF-C, is a ligand for the Flt4 (VEGFR-3) and KDR (VEGFR-2) receptor tyrosine kinases. EMBO J 15: 1751, 1996.

7. Mandriota SJ, Jussila L, Jeltsch M, et al: Vascular endothelial growth factor-C-mediated lymphangiogenesis promotes tumour metastasis. EMBO J 20: 672-682, 2001.

8. Stacker SA, Caesar C, Baldwin ME, et al: VEGF-D promotes the metastatic spread of tumor cells via the lymphatics. Nat Med 7: 186-191, 2001

9. Skobe M, Hawighorst T, Jackson DG, et al: Induction of tumor lymphangiogenesis by VEGF-C promotes breast cancer metastasis. Nat Med 7: 192-198, 2001.

10. Sleeman JP and Thiele W: Tumor metastasis and the lymphatic vasculature. Int J Cancer 125: 2747-2756, 2009.

11. Makinen T, Veikkola T, Mustjoki S, et al: Isolated lymphatic endothelial cells transduce growth, survival and migratory signals via the VEGF-C/D receptor VEGFR-3. EMBO J 20: 4762-4773, 2001.

12. Roberts N, Kloos B, Cassella M, et al: Inhibition of VEGFR-3 activation with the antagonistic antibody more potently suppresses lymph node and distant metastases than inactivation of VEGFR-2. Cancer Res 66: 2650-2657, 2006.

13. Shimizu K, Kubo H, Yamaguchi K, et al: Suppression of VEGFR-3 signaling inhibits lymph node metastasis in gastric cancer. Cancer Sci 95: 328-333, 2004.

14. Veikkola T, Jussila L, Makinen T, et al: Signalling via vascular endothelial growth factor receptor-3 is sufficient for lymphangiogenesis in transgenic mice. EMBO J 20: 1223-1231, 2001.

15. Burton JB, Priceman SJ, Sung JL, et al: Suppression of prostate cancer nodal and systemic metastasis by blockade of the lymphangiogenic axis. Cancer Res 68: 7828-7837, 2008.

16. Alitalo K and Carmeliet P: Molecular mechanisms of lymphangiogenesis in health and disease. Cancer Cell 1: 219-227, 2002

17. Dadras SS, Paul T, Bertoncini J, et al: Tumor lymphangiogenesis: a novel prognostic indicator for cutaneous melanoma metastasis and survival. Am J Pathol 162: 1951-1960, 2003.

18. Karpanen $\mathrm{T}$ and Alitalo $\mathrm{K}$ : Lymphatic vessels as targets of tumor therapy? J Exp Med 194: F37-F42, 2001.

19. Wu KK: Inducible cyclooxygenase and nitric oxide synthase. Adv Pharmacol 33: 179-207, 1995.

20. Tsujii M, Kawano S and DuBois RN: Cyclooxygenase-2 expression in human colon cancer cells increases metastatic potential. Proc Natl Acad Sci USA 94: 3336-3340, 1997.

21. Vane JR, Mitchell JA, Appleton I, et al: Inducible isoforms of cyclooxygenase and nitric-oxide synthase in inflammation. Proc Natl Acad Sci USA 91: 2046-2050, 1994

22. Prescott SM and Fitzpatrick FA: Cyclooxygenase-2 and carcinogenesis. Biochim Biophys Acta 1470: M69-M78, 2000.

23. Oshima M, Dinchuk JE, Kargman SL, et al: Suppression of intestinal polyposis in Apc delta716 knockout mice by inhibition of cyclooxygenase 2 (COX-2). Cell 87: 803-809, 1996.

24. Fujita T, Matsui M, Takaku K, et al: Size- and invasion-dependent increase in cyclooxygenase 2 levels in human colorectal carcinomas. Cancer Res 58: 4823-4826, 1998.
25. Rozic JG, Chakraborty C and Lala PK: Cyclooxygenase inhibitors retard murine mammary tumor progression by reducing tumor cell migration, invasiveness and angiogenesis. Int J Cancer 93: 497-506, 2001.

26. Tsujii M, Kawano S, Tsuji S, Sawaoka H, Hori M and DuBois RN: Cyclooxygenase regulates angiogenesis induced by colon cancer cells. Cell 93: 705-716, 1998.

27. Williams CS, Tsujii M, Reese J, Dey SK and DuBois RN: Host cyclooxygenase-2 modulates carcinoma growth. J Clin Invest 105: $1589-1594,2000$.

28. Leahy KM, Ornberg RL, Wang Y, Zweifel BS, Koki AT and Masferrer JL: Cyclooxygenase-2 inhibition by celecoxib reduces proliferation and induces apoptosis in angiogenic endothelial cells in vivo. Cancer Res 62: 625-631, 2002.

29. Masferrer JL, Leahy KM, Koki AT, et al: Antiangiogenic and antitumor activities of cyclooxygenase- 2 inhibitors. Cancer Res 60: 1306-1311, 2000

30. Takahashi K, Kanazawa, Akiyama et al: Establishment and characterization of a cell line (SAS) from poorly differentiated human squamous cell carcinoma of the tongue. J Jpn Stomatol Soc 38: 20-28, 1989.

31. Shintani S, Mihara M, Nakahara Y, et al: Lymph node metastasis of oral cancer visualized in live tissue by green fluorescent protein expression. Oral Oncol 38: 664-669, 2002.

32. Iwata C, Kano MR, Komuro A, et al: Inhibition of cyclooxygenase-2 suppresses lymph node metastasis via reduction of lymphangiogenesis. Cancer Res 67: 10181-10189, 2007.

33. Hida T, Yatabe Y, Achiwa $\mathrm{H}$, et al: Increased expression of cyclooxygenase 2 occurs frequently in human lung cancers, specifically in adenocarcinomas. Cancer Res 58: 3761-3764, 1998.

34. Ristimaki A, Honkanen N, Jankala H, Sipponen P and Harkonen M: Expression of cyclooxygenase-2 in human gastric carcinoma. Cancer Res 57: 1276-1280, 1997.

35. Sano H, Kawahito Y, Wilder RL, et al: Expression of cyclooxygenase- 1 and -2 in human colorectal cancer. Cancer Res 55: 3785-3789, 1995

36. Zimmermann KC, Sarbia M, Weber AA, Borchard F, Gabbert HE and Schror K: Cyclooxygenase-2 expression in human esophageal carcinoma. Cancer Res 59: 198-204, 1999.

37. Choe MS, Zhang X, Shin HJ, Shin DM and Chen ZG: Interaction between epidermal growth factor receptor- and cyclooxygenase 2-mediated pathways and its implications for the chemoprevention of head and neck cancer. Mol Cancer Ther 4: 1448-1455, 2005.

38. Mendes RA, Carvalho JF and Waal I: An overview on the expression of cyclooxygenase-2 in tumors of the head and neck. Oral Oncol 45: e124-e128, 2009.

39. Da MX, Wu XT, Wang J, et al: Expression of cyclooxygenase-2 and vascular endothelial growth factor-C correlates with lymphangiogenesis and lymphatic invasion in human gastric cancer. Arch Med Res 39: 92-99, 2008.

40. Su JL, Shih JY, Yen ML, et al: Cyclooxygenase-2 induces EP1and HER-2/Neu-dependent vascular endothelial growth factor-C up-regulation: a novel mechanism of lymphangiogenesis in lung adenocarcinoma. Cancer Res 64: 554-564, 2004.

41. Zhang XH, Huang DP, Guo GL, et al: Coexpression of VEGF-C and COX-2 and its association with lymphangiogenesis in human breast cancer. BMC Cancer 8: 4, 2008.

42. Di JM, Zhou J, Zhou XL, et al: Cyclooxygenase-2 expression is associated with vascular endothelial growth factor-C and lymph node metastases in human prostate cancer. Arch Med Res 40: 268-275, 2009.

43. Oka M, Iwata C, Suzuki HI, et al: Inhibition of endogenous TGF-beta signaling enhances lymphangiogenesis. Blood 111: 4571-4579, 2008.

44. Zwaans BM and Bielenberg DR: Potential therapeutic strategies for lymphatic metastasis. Microvasc Res 74: 145-158, 2007.

45. Burton JB, Johnson M, Sato M, et al: Adenovirus-mediated gene expression imaging to directly detect sentinel lymph node metastasis of prostate cancer. Nat Med 14: 882-888, 2008

46. Kalluri R and Weinberg RA: The basics of epithelial-mesenchymal transition. J Clin Invest 119: 1420-1428, 2009.

47. Wendt MK, Allington TM and Schiemann WP: Mechanisms of the epithelial-mesenchymal transition by TGF-beta. Future Oncol 5: $1145-1168,2009$. 